\title{
Bartter Syndrome: A Rare Renal Tubulopathy Presenting with Polyuria, Generalized Weakness and Recurrent Tetany in an Adolescent Male
}

\author{
Satyajit-Mallick, Hasanat MA, Fariduddin M, Tahseen- Mahmood, Mohona-Zaman, Sharmin-Jahan* \\ Department of Endocrinology, Bangabandhu Sheikh Mujib Medical University (BSMMU), Dhaka, Bangladesh \\ *Corresponding author: sharmindmc@yahoo.com
}

\begin{abstract}
Bartter syndrome is a rare autosomal recessive renal tubulopathy resulting in hypokalemic, hypochloremic metabolic alkalosis with hyperreninemia. It consists of a set of closely related overlapping syndromes having variable presentation. Advances in molecular diagnostics have revealed the underlying mutations in numerous genes that affect the function of ion channels and transporters which normally mediate salt reabsorption in the distal nephron segments. The classic manifestations are childhood onset of fatigue, polyuria, polydipsia, salt craving, vomiting, dehydration, short stature, and failure to thrive. It should be suspected in any young, normotensive individual presenting with persistent hypokalemic alkalosis in the absence of surreptitious diuretic abuse. It requires high index of clinical suspicion for appropriate diagnosis and timely treatment have been shown to reverse the clinical and biochemical abnormalities thereby improve the outcome. Here we report a 19-year-old boy with classical Bartter syndrome that was treated with Potassium supplementation, NSAID, spironolactone and he showed significant improvement on subsequent follow-up.
\end{abstract}

Keywords: hypokalemia, tubulopathy, Bartter syndrome, Gietlman syndrome

Cite This Article: Satyajit-Mallick, Hasanat MA, Fariduddin M, Tahseen- Mahmood, Mohona-Zaman, and Sharmin-Jahan, "Bartter Syndrome: A Rare Renal Tubulopathy Presenting with Polyuria, Generalized Weakness and Recurrent Tetany in an Adolescent Male.” American Journal of Medical Case Reports, vol. 6, no. 7 (2018): 124-127. doi: 10.12691/ajmcr-6-7-1.

\section{Introduction}

Bartter syndrome represents a set of closely related, autosomal recessive renal tubular disorders characterized by hypokalemia, hypochloremia, metabolic alkalosis, and hyperreninemia with normal blood pressure resulting into excessive urinary losses of sodium, chloride, and potassium. Although rare, Bartter's syndrome in mildest form may go unrecognized for a long period.

\section{Case Presentation}

A 19-year-old student was admitted with increased frequency of micturition since 4 years of age and generalized weakness and fatigue for last 6 years. He was the only issue of consanguineous marriage, born at full term with normal birth weight having no significant ante-natal or post natal event .He stated increased frequency of micturition 8 to 10 times per day, relatively more frequent at night associated with dry mouth and polydipsia without any documentation of urine volume. His weakness was of gradual onset without any diurnal variation, often aggravated by excessive physical activity and relieved partially by rest, fruits, oral saline. It was associated with muscle cramps and pain but not with excessive sweating, palpitation, heat intolerance, carbohydrate rich meal intake, diarrhoea or vomiting. He had experienced recurrent episodes of involuntary painful spasms of hands and feet for last 3 years associated with perioral paresthesia, tingling and numbness of hands and feet .Interestingly each episode tend to be relieved by taking milk. He was unable to attain adequate physical growth in comparison to his peers though having normal nutritional history, absence of chronic diarrhoea or any other significant systemic disease. His school performance was satisfactory. After consultation with a general physician he was investigated and found to have persistent hypokalemia $(<3 \mathrm{mmol} / \mathrm{L})$ and then prescribed to have Potassium syrup and oral saline without any significant symptomatic or biochemical improvement. After that he consulted at a tertiary care OPD where he was investigated and found to have hypokalemia, mildly raised serum creatinine and bilateral renal calculi with early parenchymal disease (Table 1). Distal renal tubular acidosis resulting from Sjogren's syndrome was suspected on the basis of dry mouth along with these clinical and biochemical abnormalities. His subsequent reports revealed hypocalcemia, hypomagnesemia and high PTH (Table 1). 24 hours urine collection for electrolytes revealed all electrolytes in higher than normal reference value with high 24 hours urinary volume and urinary $\mathrm{P}^{\mathrm{H}}-6.87$ (Table 2). Interestingly arterial blood gas analysis showed alkaline venous plasma $\left(\mathrm{P}^{\mathrm{H}} 7.529\right)$; plasma bicarbonate was 31mmol/l (24-28 mmol/l). 
After all these, the patient was referred to Endocrinology department of BSMMU.

So,a normotensive, hypokalemic young male presented with muscle weakness, polyuria, recurrent tetany having high urinary electrolyte excretion but low normal serum calcium, high PTH, low serum magnesium along with bilateral renal calculi, mild renal impairment and alkaline venous plasma led us to think about any surreptious diuretic abuse or renal tubulopathies. On review, there was no history or evidence of diuretics or related drug abuse, maternal polyhydramnios, loin pain, dysuria or similar illness in his family. General physical examination revealed weight-35 kg, height- $154 \mathrm{~cm}$, BMI- $14.8 \mathrm{~kg} / \mathrm{m}^{2}$ without any pallor or icterus, there was no sign of dehydration, BP was 90/70 mm Hg without any postural drop, other vital parameters were normal, thyromegaly or lymphadenopathy was absent (Figure 1). However both Chvostek's and Trosseau's signs were positive (Figure 2). His systemic examination was non-contributory except for the diminished muscle tone and muscle power (grade 4).

Repetition of previous reports showed persistent electrolyte abnormalities as expected, in addition he had high normal alkaline phosphatase and Vit-D in the insufficient range with raised urinary electrolye excretion (Table 1, Table 2). His arterial blood gas analysis was done which showed metabolic alkalosis- $\mathrm{P}^{\mathrm{H}}$ : 7.48; $\mathrm{PCO}_{2}$ : $35 \mathrm{~mm} \mathrm{Hg} ; \mathrm{PO}_{2}$ : $101 \mathrm{~mm} \mathrm{Hg} ; \mathrm{HCO}_{3}{ }^{-} 26.6 \mathrm{mmol} / \mathrm{L} ; \mathrm{SPO}_{2}$ : 98.3\%. Serum aldosterone was elevated-256.10 pg/ml [ref. value-20-180 pg/ml] and plasma renin was low normal $-6.05 \mathrm{pg} / \mathrm{ml}$ [ref. value-4.0-37.52 pg/ml]. In this context our diagnosis was Bartter syndrome with secondary hyperparathyroidism and bilateral nephrocalcinosis. Genetic study could not be done due to non-availability of such specialized laboratories in our country and for financial constraints.

He was managed accordingly with potassium enriched fruits and intravenous potassium followed by oral supplement, tab. Spironolctone 25mg thrice daily, tab Indomethacin 25mg thrice daily, tab Calcium carbonate 1 gram daily. On follow-up at 2 weeks his urine volume reduced to initially $2000 \mathrm{ml} / 24$ hours and then to normal volume of $1000-1400 \mathrm{ml} / 24$ hours while electrolytes also became normal with serum potassium $3.8 \mathrm{mmol} / \mathrm{L}$, serum sodium $136 \mathrm{mmol} / \mathrm{l}$ and, serum calcium $8.9 \mathrm{mg} / \mathrm{dl}$, serum creatinine also dropped down to $1.2 \mathrm{mg} / \mathrm{dl}$. However he developed mild, tender gynecomastia that was treated conservatively and ultimately resolved on continuation of treatment. At present he is on regular follow-up showing remarkable clinical as well as biochemical improvement.

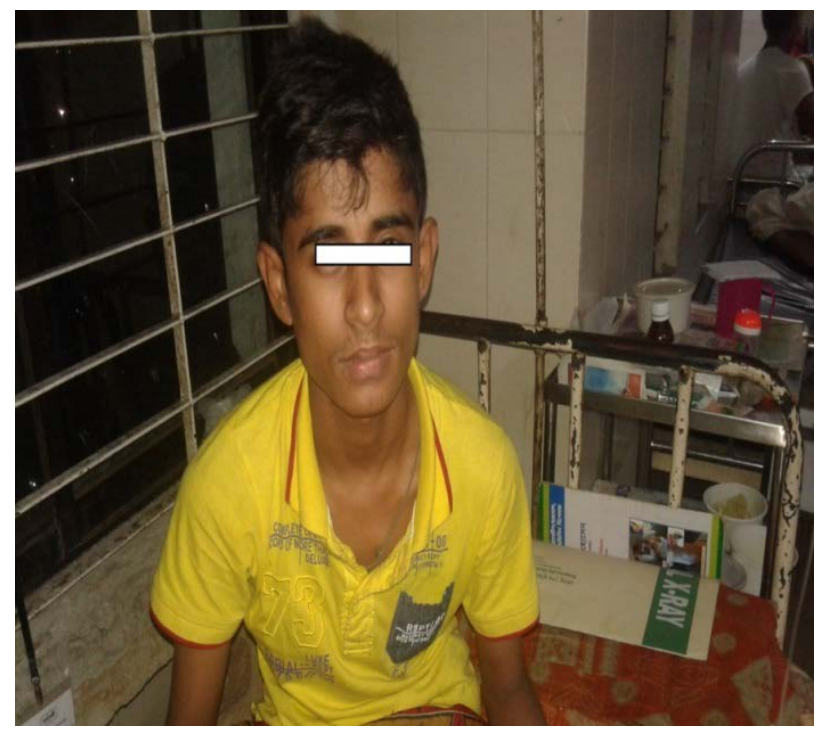

Figure 1. Front profile of the patient

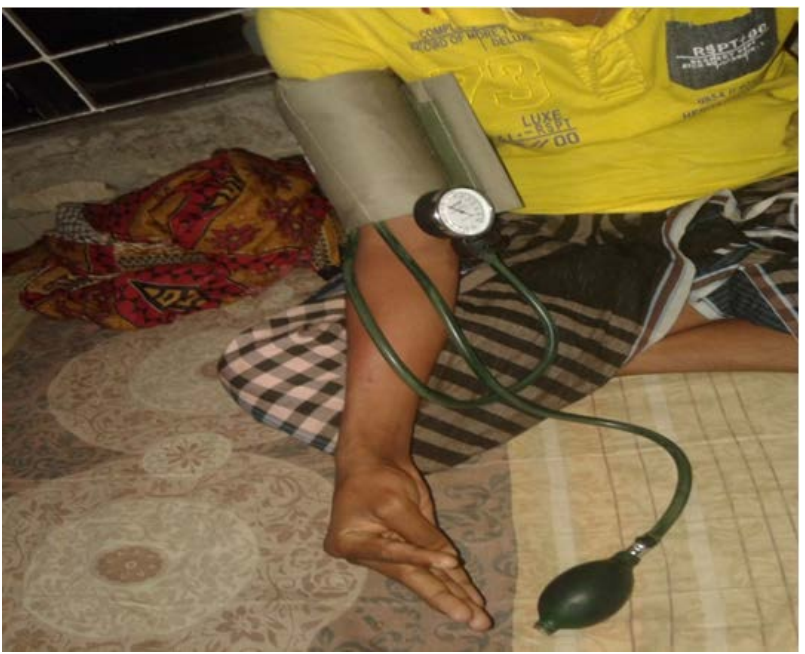

Figure 2. Positive Trousseau's sign in the patient

Table 1. Biochemical report of the patient

\begin{tabular}{|l|c|c|c|}
\hline \multirow{2}{*}{ Biochemical parameter } & \multicolumn{2}{|c|}{ Obtained value } & \multirow{2}{*}{ Reference value } \\
\cline { 2 - 3 } & $1^{\text {st }}$ occasion & $2^{\text {nd }}$ occasion & $0.7-1.2 \mathrm{mg} / \mathrm{dl}$ \\
\hline Serum creatinine & $1.49 \mathrm{mg} / \mathrm{dl}$ & $1.52 \mathrm{mg} / \mathrm{dl}$ & $3.5-5.2 \mathrm{mmol} / \mathrm{l}$ \\
\hline Serum Potassium & $1.9 \mathrm{mmol} / \mathrm{l}$ & $2.16 \mathrm{mmol} / \mathrm{l}$ & $2.12-2.62 \mathrm{mmol} / \mathrm{l}$ \\
\hline serum calcium & $2.19 \mathrm{mmol}$ & $2.11 \mathrm{mmol} / \mathrm{l}$ & $0.65-1.05 \mathrm{mmol} / \mathrm{l}$ \\
\hline serum magnesium & $0.64 \mathrm{mmol} / \mathrm{l}$ & $0.68 \mathrm{mmol} / \mathrm{l}$ & $1.59-7.23 \mathrm{mmol} / \mathrm{l}$ \\
\hline PTH & $13.64 \mathrm{mmol} / \mathrm{L}$ & $16.6 \mathrm{~m} \mathrm{~mol} / \mathrm{l}$ & $46-116 \mathrm{U} / \mathrm{l}$ \\
\hline Alkaline phosphatase & & $115 \mathrm{U} / \mathrm{l}$ & $<30 \mathrm{ng} / \mathrm{ml}-$-Insufficiency \\
\hline Vit-D & & $28.5 \mathrm{ng} / \mathrm{ml}$ & \\
\hline
\end{tabular}

Table 2. Biochemical parameter of 24-hour urinalysis of the patient

\begin{tabular}{|c|c|c|c|}
\hline \multirow{2}{*}{ Biochemical parameter } & \multicolumn{2}{|c|}{ Patient's value } & \multirow{2}{*}{ Reference value } \\
\cline { 2 - 3 } & $1^{\text {st }}$ occasion & $2^{\text {nd }}$ occasion & $800-1800 \mathrm{ml}$ \\
\hline 24 hours urinary volume & $3709 \mathrm{ml}$ & $5500 \mathrm{ml}$ & $4.6-8.0$ \\
\hline uriary $\mathrm{P}^{\mathrm{H}}$ & 6.87 & & $<20 \mathrm{mmol} / \mathrm{day}$ \\
\hline urinary sodium & $137.97 \mathrm{mmol} / \mathrm{day}$ & & $<20 \mathrm{mmol} / \mathrm{day}$ \\
\hline urinary potassium & $43.77 \mathrm{mmol} / \mathrm{day}$ & $76.45 \mathrm{mmol} / 24 \mathrm{hrs}$ & $<40 \mathrm{mmol} / 24 \mathrm{hrs}$ \\
\hline urinary chloride & $158.75 \mathrm{mmol} / \mathrm{day}$ & $429 \mathrm{mmol} / 24 \mathrm{hrs}$ & $<7.5 \mathrm{mmol} / \mathrm{l}$ \\
\hline urinary calcium & $10.52 \mathrm{mmol} / \mathrm{day}$ & $9.76 \mathrm{mmol} / \mathrm{day}$ & \\
\hline
\end{tabular}




\section{Discussion}

Bartter syndrome, originally described by Bartter and colleagues in 1962 [1], represents a set of closely related, autosomal recessive renal tubular disorders characterized by hypokalemia, hypochloremia, metabolic alkalosis, and hyperreninemia with normal blood pressure. [2] The underlying renal abnormality results in excessive urinary losses of sodium, chloride, and potassium.

Bartter syndrome has traditionally been classified into three main clinical variants, as follows:

- Neonatal (or antenatal) Bartter syndrome

- Classic Bartter syndrome

- Gitelman syndrome.

Advances in molecular diagnostics have revealed that Bartter syndrome results from mutations in numerous genes that affect the function of ion channels and transporters which normally mediates salt reabsorption in the distal nephron segments [3,4].

Neonatal Bartter syndrome (types 1 and 2) usually presents in the newborn period. There is often preceding maternal polyhydramnios due to fetal polyuria and premature birth.Massive polyuria with life-threatening volume depletion and poor weight gain are seen. Nephrocalcinosis is universal in these types. The Classic Bartter syndrome (type 3) phenotype is highly variable. The presentation may be like the typical 'neonatal' variant that manifests in the early neonatal period or the 'classic' variant that is characterized by childhood onset of fatigue, polyuria, polydipsia, salt craving, vomiting, dehydration, short stature, and failure to thrive; nephrocalcinosis is absent and hypercalciuria is less severe/absent. Bartter syndrome type 4 is associated with sensorineural deafness. In contrast, patients with Gitelman syndrome have low serum magnesium levels with hypocalciuria with absence of signs of overt volume depletion [5].

Bartter and Gitelman syndromes are renal tubular saltwasting disorders in which the kidneys cannot reabsorb chloride in the thick ascending part of Loop of Henle (TALH) and the distal convoluted tubule (DCT) respectively, depending on the mutation [6].

Chloride is passively absorbed along most of the proximal tubule but is actively transported in the TALH and the DCT. Failure to reabsorb chloride results in a failure to reabsorb sodium and leads to excessive sodium and chloride (salt) delivery to the distal tubules, leading to excessive salt and water loss from the body [6].

The renin-angiotensin-aldosterone system (RAAS) is a feedback system activated with volume depletion. Longterm stimulation may lead to hyperplasia of the juxtaglomerular complex leading to increased synthesis of renin and aldosterone.

Angiotensin II (ANG II) is directly vasoconstrictive, increasing systemic and renal arteriolar constriction, which helps to prevent systemic hypotension. It directly increases proximal tubular sodium reabsorption.ANG II-induced renal vasoconstriction, along with potassium deficiency, produces a counter-regulatory rise in vasodilating prostaglandin E (PGE) levels. High PGE levels are associated with growth inhibition in children. In our case, the patient's growth was stunted.

High levels of aldosterone also enhance potassium and hydrogen exchange for sodium. Excessive intracellular hydrogen ion accumulation is associated with hypokalemia and intracellular renal tubule potassium depletion. This is because hydrogen is exchanged for potassium to maintain electrical neutrality. It may lead to intracellular citrate depletion, because the alkali salt is used to buffer the intracellular acid and then lowers urinary citrate excretion. Hypocitraturia is an independent risk factor for renal stone formation. The index patient is normotensive as classical Bartter's syndrome with typical characteristic bilateral nephrocalcinosis [6].

Excessive distal sodium delivery increases distal tubular sodium reabsorption and exchange with the electrically equivalent potassium or hydrogen ion. This, in turn, promotes hypokalemia, while lack of chloride reabsorption promotes inadequate exchange of bicarbonate for chloride, and the combined hypokalemia and excessive bicarbonate retention lead to metabolic alkalosis. Our patient had typical metabolic alkalosis with associated persistent hypokalemia.

Persons with Bartter syndrome often have hypercalciuria. Normally, reabsorption of the negative chloride ions promotes a lumen-positive voltage, driving paracellular positive calcium and magnesium absorption. Continued reabsorption and secretion of the positive potassium ions into the lumen of the TALH also promotes reabsorption of the positive calcium ions through paracellular tight junctions. Dysfunction of the TALH chloride transporters prevents urine calcium reabsorption in the TALH. Excessive urine calcium excretion may be one factor in the nephrocalcinosis observed in these patients. Persistently raised urinary calcium may be a cause of bilateral nephrocalcinosis in our case [6].

Calcium is usually reabsorbed in the DCT. Theoretically, chloride is reabsorbed through the thiazide-sensitive sodium chloride co-transporter and transported from the cell through a basolateral chloride channel, reducing intracellular chloride concentration. The net effect is increased activity of the voltage-dependent calcium channels and enhanced electrical gradient for calcium reabsorption from the lumen.

In Gitelman syndrome, dysfunction of the sodium chloride cotransporter (NCCT) leads to hypocalciuria and hypomagnesemia. In the last several years, the understanding of magnesium handling by the kidney has improved and advances in genetics have allowed the differentiation of a variety of magnesium-handling mutations.

While patients the variants that make up Bartter syndrome may or may not have hypomagnesemia, this condition is pathognomonic for Gitelman syndrome. The mechanism of the impaired magnesium reabsorption is still unknown; studies in NCCT knockout mice demonstrate increased apoptosis of DCT cells, which would then lead to diminished reabsorptive surface area. [7] The index patient has also hypomagesemia on each occasion of electrolytes measurement.

Clinical picture supplemented by typical electrolyte abnormality is usually sufficient for the diagnosis. However renal biopsy may be done and histologic findings may be useful in confirming the diagnosis of Bartter syndrome. In neonatal and classic Bartter syndrome, the cardinal finding is hyperplasia of the juxtaglomerular apparatus. Less frequently, hyperplasia of the medullary interstitial cells is present. Glomerular hyalinization, apical vacuolization of 
the proximal tubular cells, tubular atrophy, and interstitial fibrosis may be present as a consequence of chronic hypokalemia.

In our case, with an apparently uneventful early life the onset of symptoms was at 4 years of age with polydipsia, polyuria which are classical pointers to tubular disorders. We excluded Bartter syndrome types 1 and 2 based on the age of onset and severity; type 4 was excluded as the patient had no hearing problem. Gitelman syndrome was excluded by persistent hypercalciuria resulting into bilateral nephrocalcinosis . Actual type of the disease could not be detected as in type 3 Bartter, there should not be nephrocalcinosis. Plasma aldosterone level was typically high but plasma renin level was at low normal along with low blood pressure.

Correction of dehydration and electrolytic imbalance is the mainstay of treatment of Bartter syndrome. Apart from potassium supplementation, administration of non-steroidal anti-inflammatory drugs (NSAIDs) is useful as the effects of prostaglandin synthetase inhibition include an increase in the plasma potassium concentration (however, this rarely exceeds $3.5 \mathrm{mEq} / \mathrm{L}$ ) with a decrease in the magnitude of polyuria, and improved general well-being. [8] Indomethacin at a dose of $1-5 \mathrm{mg} / \mathrm{kg} / \mathrm{day}$ is most frequently used and well-tolerated. Other drugs used are acetylsalicylic acid, ibuprofen, or ketoprofen. Mild renal impairment was another challenge for management with NSAID in our case. Addition of potassium sparing diuretics may be initially effective in the control of hypokalemia but their effect is transient. As it is an autosomal recessive disorder, genetic counseling should be offered to couples whose previous siblings have suffered from the disorder. [9] Our patient was the only son of his parent.

The degree of disability depends on the severity of the receptor dysfunction, but the prognosis in many cases is good, most able to lead fairly normal lives. Neither Bartter nor Gitelman syndrome is curable. With treatment, plasma renin and aldosterone levels normalize. Timely therapy improves the patient's clinical condition and allows catch-up growth. Bone age is usually appropriate for chronological age, and pubertal and intellectual development are normal with treatment. The effectiveness of long-term use of prostaglandin synthetase inhibitors is well established. Some patients may experience a recurrence of hypokalemia, which can be managed by adjusting the indomethacin dose or with potassium supplementation. The disease does not recur in the patient with a transplanted kidney [10]. Early diagnosis and proper treatment of Bartter's would prevent tubulointerstitial nephritis and renal failure. [11,12] In this patient, mild nonspecific symptoms, signs, and clinical rarity of Bartter syndrome perplexed the physicians that led to delayed diagnosis.

\section{Conclusion}

Recurrent or persistent hypokalemia in a young, normotensive person in the absence of surreptitious diuretic or laxative abuse should arouse the possibility of Barrter syndrome. High index of clinical suspicion is required for timely diagnosis and judicious treatment has been shown to improve the prognosis and prevent various complications.

\section{Conflict of Interest}

The authors declare that there is no conflict of interest regarding the publication of this paper.

\section{References}

[1] Bartter FC, Pronove P, Gill JR. Hyperplasia of the juxtaglomerular complex with hyperaldosteronism and hypokalemic alkalosis. American Journal of Medicine. 1962. 33: 811-828.

[2] Bokhari SRA, Mansur A. Bartter Syndrome. 2017 Jun. [Medline]. [Full Text].

[3] Lin SH, Yang SS, Chau T. A practical approach to genetic hypokalemia. Electrolyte Blood Press. 2010 Jun. 8(1): 38-50. [Medline]. [Full Text].

[4] Devarajan P. Bartter syndrome: e-Medicine. October 2008. Available at http://www.emedicine.com/PED/topic210.htm.

[5] Brenner B, Levine S. Inherited disorders of renal tubule. In: Brenner and Rector's The kidney. 8th ed. Philadelphia, PA: WBSaunders; 2007:1411-1414 [chapter 40].

[6] Frassetto LA. Bartter syndrome. e-Medicine from Web MD. November 2017. Available at http://emedicine.medscape.com/article/238670-pathophysiology.

[7] Chen YH, Lin JJ, Jeansonne BG, et al. Analysis of claudin genes in pediatric patients with Bartter's syndrome. Ann N Y Acad Sci. 2009 May. 1165: 126-34.

[8] Proesmans W, Massa G, Vanderschueren-Lodeweyckx M. Growth from birth to adulthood in a patient with theneonatal form of Bartter syndrome. Pediatr Nephrol.1988; 2: 205-209.

[9] Muranjan MN, Kantharia VC, Bavdekar SB, Kabde U, Parmar RC. Neonatal Bartter syndrome. Indian J Pediatr. 2002; 69: 105-107.

[10] Chaudhuri A, Salvatierra O Jr, Alexander SR, Sarwal MM. Option of pre-emptive nephrectomy and renal transplantation for Bartter's syndrome. Pediatr Transplant. 2006 Mar. 10(2): 266-70.

[11] Heffernan A, Steffensen TS, Gilbert-Barness E, Perlman S. Bartter syndrome presenting as poor weight gain and abdominal mass in an infant. Fetal Pediatr Pathol. 2008; 27: 232-243.

[12] Walsh SB, Unwin E, Vargas-Poussou R, Houillier P, Unwin R. Does hypokalaemia cause nephropathy? An observational study of renal function in patients with Bartter or Gitelman syndrome. QJM. 2011 Nov. 104(11): 939-44 